\title{
Magnetoresistance through spin-polarized p states
}

\author{
Nikos Papanikolaou \\ Institute of Materials Science, NCSR 'Demokritos', GR-15310, Ag Paraskevi Attiki, Greece \\ E-mail: papanikolaou@ims.demokritos.gr
}

Received 10 April 2003

Published 11 July 2003

Online at stacks.iop.org/JPhysCM/15/5049

\begin{abstract}
We present a theoretical study of the ballistic magnetoresistance in Ni contacts using first-principles, atomistic, electronic structure calculations. In particular we investigate the role of defects in the contact region with the aim of explaining the recently observed spectacular magnetoresistance ratio. Our results predict that the possible presence of spin-polarized oxygen in the contact region could explain conductance changes by an order of magnitude. Electronic transport essentially occurs through spin-polarized oxygen $\mathrm{p}$ states, and this mechanism gives a much higher magnetoresistance than that obtained assuming clean atomically sharp domain walls alone.
\end{abstract}

Achieving an understanding of spin transport in order to apply it in electronic devices has been an intensively pursued goal over the last few years. In this direction, spectacular effects have been reported in electrodeposited Ni nanocontacts where the resistance changes by two to three orders of magnitude on applying a small magnetic field at room temperature $[1,2]$. This followed after effort was focused on the magnetoresistance effect of electrodeposited nanocontacts $[3,4]$. Smaller magnetoresistance ratios, up to few hundred per cent, were reported for ballistic $\mathrm{Ni}, \mathrm{Co}$, and $\mathrm{Fe}$ nanoconstrictions [5] and $\mathrm{Fe}_{3} \mathrm{O}_{4}$ nanocontacts [6]. There have been several attempts to explain the experimental data. One possibility considered is the scattering of the electrons in a domain wall [5-8]. The width of a domain wall in a nanocontact is predicted to be of the order of the nanocontact size [9], and such a reduction of the domain wall width increases the magnetoresistance. Magnetostriction effects could also lead to an increased magnetoresistance [6], but most probably cannot explain the experiments [3]. Alternatively, a thin domain wall pinned in a magnetic dead layer together with the possibility of $100 \%$ polarization of the Ni d electrons was also proposed as a possible explanation [2,3]. Moreover, the effect of several ballistic nanocontacts in parallel requires further study, as pointed out in the most recent work in [1].

There has been some experimental effort to measure the magnetoresistance due to a domain wall in a constriction [10-13], but all studies found magnetoresistance values of the order of $1 \%$ or less. This result is not far from the theoretical predictions of Levy and Zhang [14], 
who reported values between 2 and $11 \%$. Ab initio calculations for the ballistic conductance through an atomically sharp domain wall [15] predict MR ratios up to $60-70 \%$, which is at least one order of magnitude lower than the experimental values for the electrodeposited Ni nanocontacts. On the other hand, phenomenological models $[6,8]$ seem to account for magnetoresistance values up to a few hundred per cent, by assuming domain wall scattering. However, it is difficult to assign a realistic spin polarization in explaining magnetoconductance (MC) values of the order of thousands of per cent.

Since the large effects appear for electrodeposited $\mathrm{Ni}$ nanocontacts [4], the electrodeposition process should play a decisive role in the nanocontact properties. The chemistry and structure of the contacts is not yet clear, but there is some indication that the diameter of the nanocontacts is not the crucial factor for obtaining large magnetoresistances [2]. Generally, different conductance values are attributed to different diameters of the contact area; however, the transport mechanism is still not clear.

In this work we propose a new mechanism, based on the presence of spin-polarized oxygen in the contact region, that could explain the high magnetoresistance values in $\mathrm{Ni}$ contacts. We considered $\mathrm{Cl}, \mathrm{S}, \mathrm{O}$, and $\mathrm{C}$ in the contact region [2], and studied the influence of the nanocontact chemistry on the magnetotransport properties by means of electronic structure calculations. Only oxygen was found to have a magnetic moment in the structures that we studied, while the presence of a magnetic dead layer cannot explain the observed effect since it reduces the magnetoresistance ratio, and this is confirmed by our calculations.

We model the nanocontacts by two semi-infinite $\mathrm{Ni}(001)$ ideal leads covered with a full monolayer (ML) of $\mathrm{Cl}, \mathrm{S}, \mathrm{C}$, and $\mathrm{O}$, and bring them close together. We use the experimental lattice constant of $\mathrm{Ni}: a_{0}=6.65$ au. The distance of the defect layer from the Ni surface is $a_{0} / 2$ and the defect layers are separated by $a_{0}$. We do not intend to model an O overlayer on the $\mathrm{Ni}(001)$ surface; the structure and magnetic properties of $\mathrm{O}$ on $\mathrm{Ni}$ have been studied well in the past both theoretically and experimentally $[16,17]$, and are not relevant to the structure of electrodeposited Ni nanocontacts. Our aim is to use a simple model structure in order to achieve the minimum requirements that demonstrate the effect of spin-polarized transport through p states. The experimental structure in the nanocontacts where the high magnetoresistance is observed is not well known, but spin polarization on the $\mathrm{O}$ atoms can occur for example if a thin $\mathrm{NiO}$ layer is present in the contact [18]. Moreover, we assume that the electronic transport is ballistic.

The electronic structure is calculated using the screened Korringa-Kohn-Rostoker(KKR) Green function method, details of which can be found elsewhere [19]. The potential is assumed to be spherically symmetric around each atom. We considered an angular momentum cutoff $l_{\max }=3$ for the wavefunctions and the Green function, and $l_{\max }=6$ in the multipole expansion of the charge density. The screening transformation of the KKR method allows for the description of semi-infinite geometries using the decimation method. Exchange and correlation effects are taken into account within the local density approximation of the density functional theory [20] using the parametrization of Vosko et al [21]. The conductance $g$ is calculated within the Kubo linear response theory as formulated by Baranger and Stone [22]. We consider a sample connected to two semi-infinite leads. Two surfaces $C_{n}$ and $C_{m}$ separate the leads from the sample. Following [22] the conductance between the two leads at energy $E$ and at zero temperature is given by

$$
g=\frac{-e^{2} \hbar^{3}}{8 \pi M^{2}} \int_{C_{n}} \mathrm{~d} S \int_{C_{m}} \mathrm{~d} S^{\prime} G^{+}\left(\boldsymbol{r}, \boldsymbol{r}^{\prime} ; E\right) \stackrel{\leftrightarrow}{\nabla} \stackrel{\leftrightarrow}{\nabla}^{\prime} G^{-}\left(\boldsymbol{r}^{\prime}, \boldsymbol{r} ; E\right)
$$

where $f(r) \stackrel{\leftrightarrow}{\nabla} g(r)=f(r) \nabla g(r)-g(r) \nabla f(r)$, and $G^{+}, G^{-}$are the advanced and retarded Green functions, respectively. The systems under consideration have a two-dimensional (2D) 

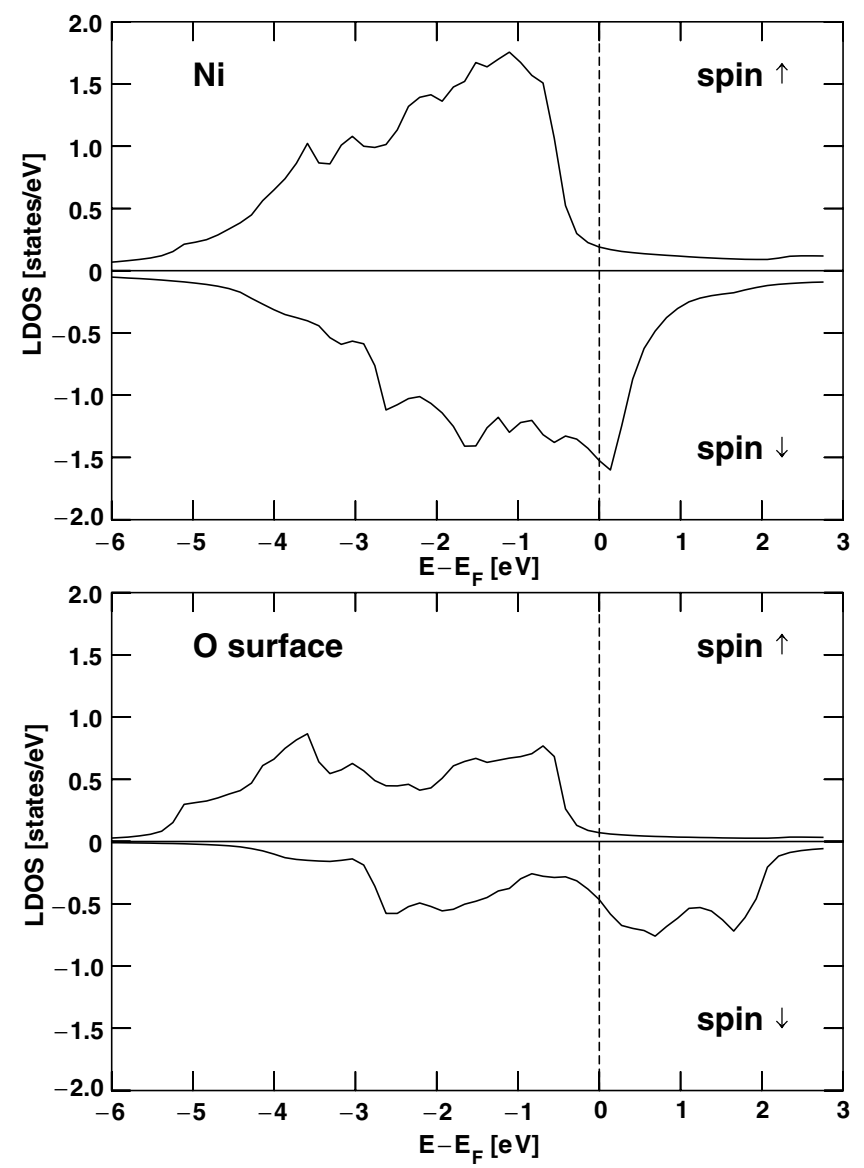

Figure 1. Spin-resolved densities of states for the interface $\mathrm{O}$ and first Ni layers for $1 \mathrm{ML}$ of oxygen on a fcc $\mathrm{Ni}(001)$ junction.

periodicity parallel to the interfaces, and the surface integrations in equation (1) are performed using a Fourier transform. A detailed presentation will be reported elsewhere [23]. The Green function for the conductance calculation was evaluated using a small imaginary part for the energy, $\operatorname{Im} E=0.02$ mRyd.

In figure 1 we present the local densities of states (LDOSs) of $\mathrm{O}$ at the interface and of $\mathrm{Ni}$ in the neighbouring layer. The magnetic moment of $\mathrm{Ni}$ is $0.85 \mu_{B}$, compared to the bulk value of $0.61 \mu_{B}$; this value is very close to the clean $\mathrm{Ni}(001)$ surface moment. The oxygen in our structure has a rather large moment of $1.4 \mu_{B}$. We note that the difference of the LDOSs at the Fermi level is larger for the Ni d states compared with the $\mathrm{p}$ states of $\mathrm{O}$, as can seen in figure 1. However, the induced $\mathrm{p}$ magnetization is the key factor for achieving high MC ratios. In figure 2 we present the conductance for each spin channel, for parallel (P) and antiparallel (AP) orientations of the magnetic moments in the Ni leads, together with the MC ratio, defined as $\mathrm{MC}=\left(g_{P}-g_{A P}\right) / g_{A P}$. The magnetic profile changes only little for the AP configuration and the $\mathrm{O}$ remains highly spin polarized. As we can see in the bottom panel of figure 2, the $\mathrm{MC}$ ratio was found to be about $450 \%$ at the Fermi level and increases to almost $700 \%$ at $0.3 \mathrm{eV}$ above $E_{F}$. The conductance and MC ratio peaks are closely related to the LDOS 

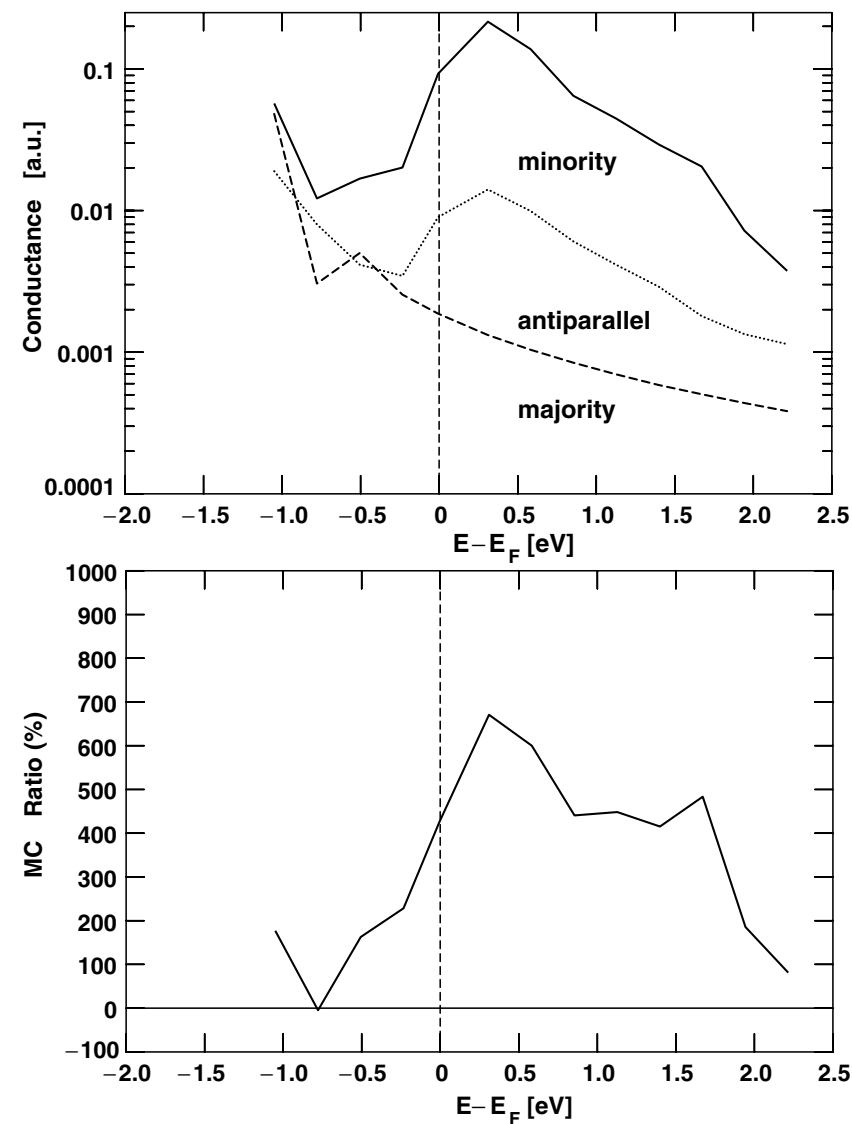

Figure 2. Top panel: conductance variation with energy for $1 \mathrm{ML}$ of oxygen on a $\mathrm{Ni}(001)$ junction, for majority-spin (full curve) and minority-spin (dashed curve) channels in the parallel spin configuration together with the AP spin configuration (dotted curve). The conductance is in arbitrary units. Bottom panel: the MC ratio for the same junction.

at the $\mathrm{O}$ site. Comparing figure 1 with 2 , we can see that since the majority-spin states are occupied (they are below $E_{F}$ ), the variation of the $\mathrm{MC}$ ratio above $E_{F}$ reflects the variation of the minority LDOS. The p polarization leads to a large contrast in conductance between majority and minority channels. Although the spin polarization of Ni at $E_{F}$ is almost twice the $\mathrm{O}$ one, it is the relatively small spin polarization of the oxygen $\mathrm{p}$ states which is responsible for the spectacular MC ratio. Indeed, our calculations show that in a pure Ni contact where two $\mathrm{Ni}(001)$ leads are separated by one vacuum layer, the $\mathrm{MC}$ ratio has a maximum value of only $\approx 70 \%$, which is similar to the values reported previously for the magnetoresistance of abrupt, atomically sharp domain walls in the ballistic regime [15]. The question of spin polarization and its relevance to the observed current spin polarization has been discussed before [24]. Our results introduce another factor, the character of the polarization, which is missing from the models used previously to explain the observed effects $[6,8]$. Spin polarization of $p$ orbitals is more difficult to produce but, once this is achieved, highly spin-polarized currents are possible. In the present work this was demonstrated with $\mathrm{O}$, but other substances might lead to similar effects as well.

In order to exclude the possibility that the large MC values are an artifact of the $2 \mathrm{D}$ periodicity, we used a $\mathrm{c}(2 \times 2)$ structure for the $\mathrm{O}$ on the surface of $\mathrm{Ni}(001)$ and recalculated 


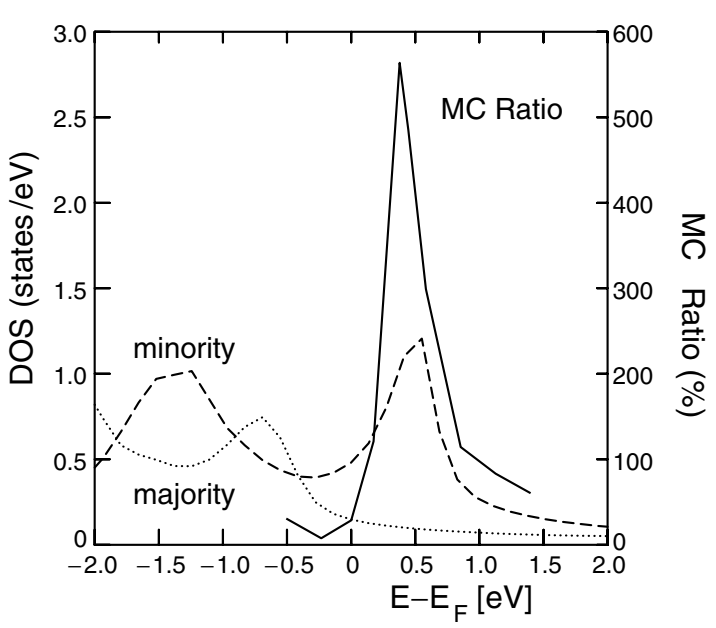

Figure 3. Spin-resolved densities of states on the $\mathrm{O}$ site for two Ni(001) leads covered with $0.5 \mathrm{ML}$ of oxygen on a $\mathrm{c}(2 \times 2)$ structure and separated by one vacuum layer. The MC ratio as a function of energy close to the Fermi level is also shown (full curve).

the transport properties. Despite the fact that $\mathrm{c}(2 \times 2)$ structure is close to the experimental structure for an $\mathrm{O}$ overlayer on $\mathrm{Ni}(001)$ [17], the distance between $\mathrm{Ni}$ and $\mathrm{O}$ that we had to use in order to achieve polarized $\mathrm{O}$ is almost double that of the experimental structure. Since transport is mainly determined by the contact region, we are interested just in the effect of spin-polarized $\mathrm{O}$ on a magnetic substrate.

Our results for the LDOS close to the Fermi level together with the MC ratio are shown in figure 3. The magnetic moment on the $\mathrm{O}$ atoms is now reduced to $0.95 \mu_{B}$, while the $\mathrm{Ni}$ moment is less affected. This is also seen in the LDOS of $\mathrm{O}$ where the minority-spin $\mathrm{p}$ states are lower in energy compared to the case of $1 \mathrm{ML}$ oxygen coverage. As we can see, the large MC effect survives also in this configuration. The MC ratio is rather small at $E_{F}$ but increases rapidly just $0.5 \mathrm{eV}$ above $E_{F}$. The largest $\mathrm{MC}$ values, of about $500 \%$, are obtained at the oxygen $\mathrm{p}$ peak position. In other words, $\mathrm{p}$ orbitals can build highly conducting channels and, if they are spin polarized, conductance in one spin channel can even be two orders of magnitude higher than that in the other spin channel. However, in the systems that we studied the $\mathrm{MC}$ ratios are limited to $700 \%$, since conductance is not so drastically reduced in the AP configuration. We note that the proposed mechanism: high current when a bond is formed and vanishingly small current when the bond is broken, in the AP configuration, cannot exclude even higher MC ratios depending on the exact structure chosen. However, it is important that the $\mathrm{O}-\mathrm{O}$ bond energy should be smaller than exchange energy so that the bond can be broken and reformed on applying a magnetic field. The interaction between the two magnetic leads should be weak enough that the domain wall is pinned at the contact region.

Despite the fact that the proposed mechanism can explain high MC values, it cannot fully account for the experimental findings. In particular, experimentally both positive and negative magnetoresistances are observed [1]; moreover, the conductance is generally large at zero magnetic field and becomes much smaller on applying a magnetic field. The influence of an antiferromagnetic $\mathrm{NiO}$ layer in the contact region requires further study, but as the present work shows, large changes in conductance can result from spin-polarized $\mathrm{O}-\mathrm{O}$ bonds.

It is important that a large magnetic moment on the $\mathrm{O}$ atoms is not a necessary condition for obtaining a large MC ratio. Only a large difference in LDOS between minority- and 
majority-spin electrons at the Fermi level is required. Moreover, Ni itself is not a necessary ingredient; any magnetic material that could support $\mathrm{O}$ in the contact region and induce spin polarization in the $\mathrm{O}$ atoms would have similar properties.

Finally, we would like to make a clear distinction between the phenomena presented here and the effect of hot spots in the conductance studied previously in ferromagnet/vacuum/ferromagnet tunnel junctions [25]. In that case, tunnelling coexists with transport through interface states, which have a transmission coefficient close to unity and are localized in a very small region of the surface Brillouin zone. However, the hot spots depend sensitively on the symmetry of the junction and require flat, ideal interfaces. Any small disorder would suppress the effect. In contrast, the large MC ratios reported here are related to spindependent transport through $\mathrm{O}-\mathrm{O}$ bonds. The 2D structure is not a crucial factor and this is also clear from the $k_{\|}$-dependent conductance where the variation is relatively smooth and a large part of the surface Brillouin zone gives significant contributions.

Summarizing, our results show that high MC ratios in Ni nanocontacts could be explained by spin-dependent defect scattering at the nanocontact. This is in agreement with the conclusion of [14] that defect scattering can enhance the magnetoresistance in a domain wall; however, the role of spin-polarized p orbitals has not been pointed out up to now, to our knowledge. We have demonstrated that if oxygen in the contact region is spin polarized, this gives rise to high $\mathrm{MC}$ ratios. This idea is not in contradiction with the idea of scattering in an atomically sharp domain wall. However, $a b$ initio ballistic transport calculations cannot explain magnetoresistances of a few hundred per cent, assuming a defect-free atomically sharp domain wall. In contrast, the transport mechanism proposed in this work can explain the spin polarization close to $100 \%$ that is required to explain the spectacular MC ratios in electrodeposited nanocontacts. We hope that our theoretical prediction will stimulate further experimental studies to clarify the role of chemistry and in particular that of $\mathrm{NiO}$ in the magnetotransport properties of nanocontacts.

\section{Acknowledgment}

I would like to thank O Cespedeo for useful correspondence.

\section{References}

[1] Chopra H D and Hua S Z 2002 Phys. Rev. B 66 020403(R) Hua S Z and Chopra H D 2003 Phys. Rev. B 67 060401(R)

[2] Wang H, Cheng H and Garcia N 2002 Preprint cond-mat/0207516

[3] Garcia N, Munoz M, Osipov V V, Ponizovskaya E V, Qian G G, Saveliev I G and Zhao Y-W 2002 J. Magn. Magn. Mater. 24092

[4] Garcia N, Rohrer H, Saveliev I G and Zhao Y-W 2000 Phys. Rev. Lett. 853053 Garcia N, Munoz M, Qian G G, Rohrer H, Saveliev I G and Zhao Y-W 2001 Appl. Phys. Lett. 794550

[5] Tatara G, Zhao Y-W, Munoz M and Garcia N 1999 Phys. Rev. Lett. 832030 Garcia N, Munoz M and Zhao Y-W 1999 Phys. Rev. Lett. 822923

Garcia N, Munoz M and Zhao Y-W 2000 Appl. Phys. Lett. 762586

[6] Versluijs J J, Bari M A and Coey J M D 2001 Phys. Rev. Lett. 87026601

[7] Chung S H, Munoz M, Garcia N, Egelhoff W F and Gomez R D 2002 Phys. Rev. Lett. 89287203

[8] Tagirov L R, Vodopyanov B P and Efetov K B 2001 Phys. Rev. B 63104428

[9] Bruno P 1999 Phys. Rev. Lett. 832425

Molyneux V A, Osipov V V and Ponizovskaya E V 2002 Phys. Rev. B 65184425

[10] Ebels U, Radulescu A, Henry Y, Piraux L and Ounadjela K 2000 Phys. Rev. Lett. 84983

[11] Rüdiger U, Yu J, Thomas L, Parkin S S P and Kent A D 1999 Phys. Rev. B 5911914

[12] Taniyama T, Nakatani I, Namikawa T and Yamazaki Y 1999 Phys. Rev. Lett. 822780

[13] Miyake K, Shigeto K, Mibu K, Shinjo T and Ono T 2002 J. Appl. Phys. 913468

[14] Levy P M and Zhang S 1997 Phys. Rev. Lett. 795110 
[15] van Hoof J B A N, Schep K M, Brataas A, Bauer G E W and Kelly P J 1999 Phys. Rev. B 59138

[16] Godby R W, Benesh G A, Haydock R and Heine V 1985 Phys. Rev. B 32655

[17] Stuckless J T, Wartnaby C E, Al-Sarraf N, Dixon-Warren S J B, Kovar M and King D A 1997 J. Chem. Phys. 1062012

[18] Erdman N, Warschkow O, Ellis D E and Marks L D 2000 Surf. Sci. 4701

[19] Papanikolaou N, Zeller R and Dederichs P H 2002 J. Phys.: Condens. Matter 142799

[20] Hohenberg P and Kohn W 1964 Phys. Rev. 136 B864

Kohn W and Sham L J 1965 Phys. Rev. 140 A1133

[21] Vosko S H, Wilk L and Nusair N 1980 Can. J. Phys. 581200

[22] Baranger H U and Stone A D 1989 Phys. Rev. B 408169

[23] Mavropoulos P, Papanikolaou N and Dederichs P H, unpublished

[24] Mazin I I 1999 Phys. Rev. Lett. 831427

[25] Wunnicke O, Papanikolaou N, Zeller R, Dederichs P H, Drchal V and Kudrnovsky J 2002 Phys. Rev. B 65 064425 